\title{
Comparison of Standards for Accessible Design between America and China
}

\author{
Ying HAN, Xu Feng TAO \\ Jinling Institute of Technology, Jiangsu Nanjing 211169, China
}

\begin{abstract}
Aiming at the accessibility design and environmental constructions of China, comparing the differences of codes for accessibility design between domestic and abroad. It's including four aspects: the difference in audience of accessible design, content of the facilities, quantitative indexes and vision and hearing accessible design. It analyzed that the early stage of our country is backward in terms of accessibility and construction, mainly based on differences in values and professional education. In the end it put forward three suggestions to improve the construction of barrier-free environment in China.
\end{abstract}

\section{Introduction}

Accessible design begins in 1950s when people start to realize the issues of the disabled in Japan, Europe and America. They found the way to get rid of the various obstacles in the surroundings of the disabled by accessible design. Sweden and Denmark have built facilities dedicated to the disabled back then. UN released international documents like Declaration on the Rights of Disabled Persons, Convention on the Rights of Persons with Disabilities. Those documents emphasized the importance of accessible design. At that time, accessible design targeted the elder and the disabled but using a way of quarantine and special treatments. With the development of humanism design concepts, accessible design seeped in our society gradually, it has became compulsory criteria in architecture and public environment design field. Hundreds of countries and districts have made law related to the disabled also regulatory and technical standards of accessible design. Nowadays the governments of all countries are still exploring and extending their connotation while building and reforming barrier-free environment. They want to extend the meaning of accessible design to make it a universal design idea which suitable for more people [1]. Even those two concepts can exchange in some standards [2].

China has entered an aging society in 21 st century; while the disabled and the elder have diversified demand compared the general population. It is architects' career requirement and social duty to figure out how to fulfill their need in city constructions and architectural environment.

\section{The current situation of accessibility legislation and environmental construction in China}

The progress about public building accessible environment constructions was slow from Reform and Opening-up to year 2000.The government mainly focus on making welfare system better. But after 2000, especially after the 2008 Beijing Olympic Games and the 2010 Shanghai EXPO were held, the government promoted the development of accessible design in several big cities of my country. And it has formed the situation that national standards are the dominant factor, but also refined by local standards. We issued Code for design on urban roads and buildings for disabled persons in 1989 first, the code revised as Codes for design on accessibility of urban roads and buildings in 2001, and it revised again as Codes for accessibility design in 2012 [3]. The codes covers the construction of accessible environment more comprehensively, quantification degree was higher with supporting atlas. It belongs to the national mandatory standards of constructions, and it went through the test of more than 10 years of construction practice. According to the MOHURD's experts, Many local governments made local standards including Hebei Province, Liaoning Province, Hubei Province, Guangdong Province, 5 provinces of the Inner Mongolia Autonomous Region, Beijing, Shanghai, Tianjin, Nanjing, Dalian, Suzhou and other 14 city and the District of Shenzhen [4]. However, the requirements of accessible design in public area, accessible streamline, vision and hearing aid equipment in public area etc. are incomplete. Our current accessible design standards made for specific types of public buildings includes Technical standards for airport passenger terminal

*Corresponding author: Email: chelsea_han@jit.edu.cn 
facilities with accessibility by individuals with disabilities and Code for design on accessibility of railway passenger station buildings. Locations which have detailed requirements of accessible design relatively referred in national and local standards mainly includes urban road, squares, bridges, tunnels, residential areas, public parking lot, public toilets, leisure buildings for the aged etc., but we don't have specific rules for other public which have large numbers of people and wide services like offices, cultural and display public buildings.

Compared to the legislation and practice of public building accessible design in western developed countries, our country's accessible design at present stage still has a lot to catch. The gap reflected in the results of accessible environment construction on the surface, but it essentially reflected in construction legislation like standards, codes and so on which related to the hardware. The accessible design system has a deep and permanent reflection on our country's level of accessible design environment for it is a significant component of standard system of construction. There is a big disparity between Chinese and Western Developed Countries' legislation and standard of accessible design from the aspects of constitution system of legislation and standard, the depth of clauses and the expression characteristics.

\section{The comparison of accessible design between Chinese and American}

This paper compared our country's standard (i.e., Codes for accessibility design published in 2012) with western developed countries (e.g., American 2010 ADA Standards for Accessible Design short for "America standard" below). There are four main differences between those two standards.

\subsection{The difference in audience of accessible design}

Our code's general provisions 1.0 .1 points out the accessible design is and we quote "urban road and buildings that guarantee the handicapped can use conveniently and safely", although "the handicapped" should include the people who have eyesight obstacles, the aged and so on, but our country's codes mainly focus on the accessible design for people who are physically handicapped rather than people who are vision and hearing handicapped.

American standards, by contrast, fully considered human primary disability types and needs in compiling concept. For example, it clearly states that "If providing accessibility in conformance with this section to individuals with certain disabilities (e.g., those who use wheelchairs) would be structurally impracticable, accessibility shall nonetheless be ensured to persons with other types of disabilities (e.g., those who use crutches or who have sight, hearing, or mental impairments) in accordance with this section." It also takes the different body size between adults and children in to considerations. Normal Principles of General Design
Policy [5] of Japan noted that "until now, the measure to achieve accessible society is focus on removing the obstacles of the aged and the handicapped rather than considering the diversity of users. We should take measures to remove the obstacles of the aged and the widespread handicapped which not considered before including the retards or people who have mental disorder, the foreigner, the children and the adults with children." The differences in the purpose of the standards and the scope of applications reflected the gaps in accessible design idea and practice between our country's inland cities and the developed countries.

\subsection{The difference in facility types of accessible design}

Our country's codes of accessible design was written by the sequence of building's composition, the clause's content is: 1. General Provisions; 2. Terms; 3. Design Requirements of Accessible Facilities; 4. Urban Road; 5. Urban Square; 6. Urban Green Space; 7. Residential District, Residential Building; 8. Public Buildings; 9. Barrier-free Renovation of Historic Buildings. The code is applicable for various types of residential buildings and public buildings but not with a strong pertinence, the clause and design atlas are separate. Also, it lacks of guidance details. For now, only a few traffic public buildings like civil aviation terminals, railway stations etc. and buildings for the aged have specific codes for accessible design.

All types of public buildings in the United States refer to all construction facilities that are newly built, constructed, renovated, purchased, occupied or rented by the State, local and individual investments, including 20 sets or more or 20 sets of temporary occupancy [6]. Including the office of scientific research, business services, catering, health care, culture memorial, play sports, hotels, transport facilities, schools, justice, and discipline, detention and punishment, houses, gardens, public land, entertainment facilities, etc.. There is corresponding administration section and codes for accessible design for almost each kind of buildings like colleges, courts, prisons and so on. It has formed the overall accessible design environment that has law to abide by. Also, American standards have the applicable scope of mandatory provisions cleared, it didn't just define the categories of applicable buildings but also includes the applicable scope of individual buildings, for instance, when it comes to accessible design of buildings' main functional area, it said "the path of travel to the altered area, including the rest rooms, telephones, and drinking fountains serving the altered area; Alterations made to provide an accessible path of travel to the altered area will be deemed disproportionate to the overall alteration when the cost exceeds $20 \%$ of the cost of the alteration to the primary function area."

American standards mainly composed by the need and the routes people choose, it also covers a lot and has a lot of details. Basically, it covers from new buildings to traffic and residential buildings, from outdoor places to buildings' component and indoor equipment, from block 
planning to special rooms and the space and details of accessible design.

Another important difference is western developed countries emphasize the importance of accessible routes. The example is that American standards have regulations about accessible routes indoor and outdoor. It said "A 'path of travel' includes a continuous, unobstructed way of pedestrian passage by means of which the altered area may be approached, entered, and exited, and which connects the altered area with an exterior approach (including sidewalks, streets, and parking areas), an entrance to the facility, and other parts of the facility." The code has illustrations of word descriptions and dimension markings to avoid the ambiguity caused by word expression. While there is no specific regulations of accessible route and the reach range in our standards. The differences are not just reflecting on ideas, it appears that the accessibility facilities constructed by the code can't form a complete accessible route which leads to the imperfection of public accessibility environment. The legal disputes caused by the imperfection will be more complex than others. The most appropriate example nowadays is the enormous blind tracks which are discontinues and unavailable for the blind constructed in many big cities according to our country's current code.
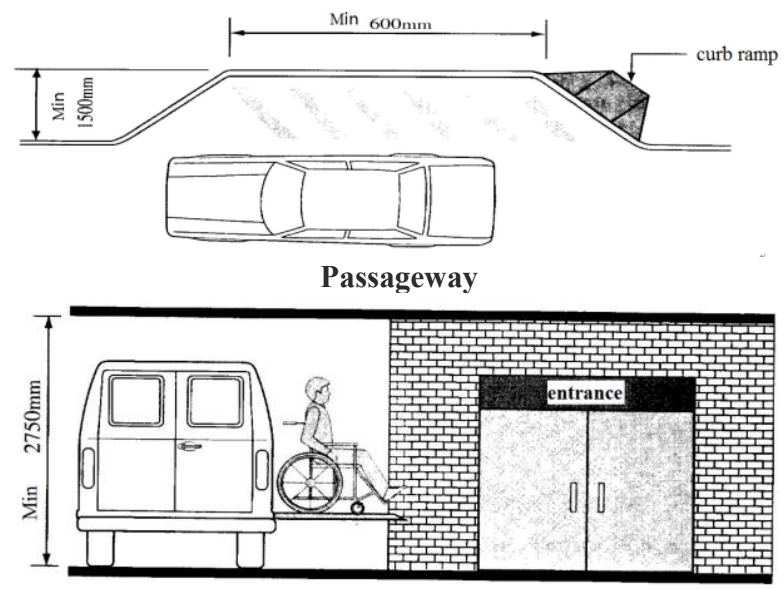

The clearance height of the passenger car area

Figure 1 Parking space access aisle and vehicle parking spaces

The control switches are massive and indispensable auxiliary facilities in modern architecture, it reflects the level of accessible design directly. But we don't have unified regulations about this, many important and frequently-used equipments are installed too high to reach. It is inconvenient not only for the man who use wheelchairs but also inconvenient for children and the aged. American standards put this part in the first place, they made regulations about control switch, door handle and door lock, window handle, faucet, electrical socket and switch, thermostat, pull fire alarm station and alarm start device. The standard said in the vicinity of the operation control space should be provided at least a $750 \mathrm{~mm} \times 1200 \mathrm{~mm}$ flat barrier-free area to facilitate the front and back and both sides close to the control switch; control switch axis height should be set from the ground

\subsection{The difference in quantitative indexes}

Compare to foreign standards, the biggest difference is we have less content of quantitative indexes in code for accessible design and standard atlas. While almost each American standards' clause has clear quantitative index or data, it also has illustrations where may cause ambiguities, it not only gives the minimum requirements but also the advisory requirements.

For example, we don't have specific rules for rugs, but in practice, rugs often become obstacles. American standards have clear regulations for the thickness of rug, Carpet or carpet tile shall have a level loop, textured loop, level cut pile, or level cut/uncut pile texture. Pile height shall be $1 / 2$ inch $(13 \mathrm{~mm})$ maximum. Exposed edges of carpet shall be fastened to floor surfaces and shall have trim on the entire length of the exposed edge.

For another example, we only set standards for plan rather than the need of space when it comes to accessibility parking lot design. But the American standards have clear regulations, it said the passageway area includes at least $2750 \mathrm{~mm}$ of clearance along the way the vehicle approaches the route or exit line and the height of the parking lot within the specified space (Fig. 1).

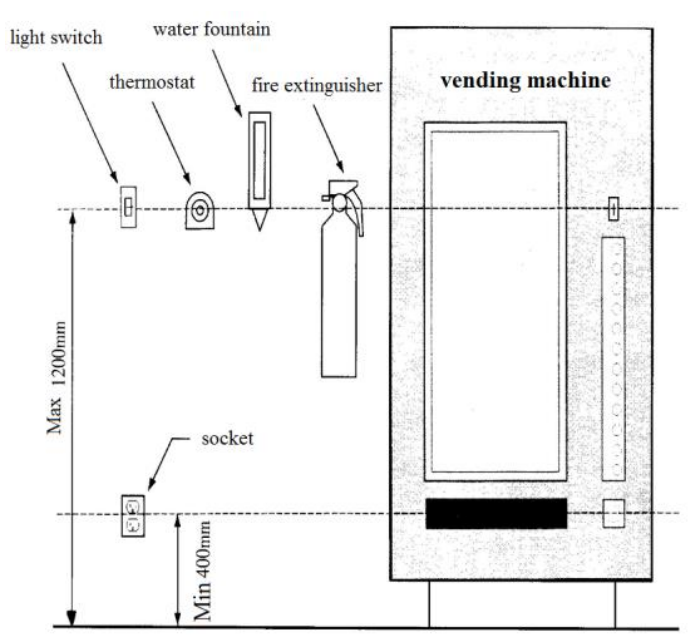

Figure 2 Height of switches

$400 \mathrm{~mm} \sim 1200 \mathrm{~mm}$ position. The control switch button should have a bulge mark or voice message (Fig. 2), indicating the function and the position of the control button.

\subsection{The difference in vision and hearing accessible design}

Our code about accessible design focus on building structure, it stresses the regulations about the handicapped that use wheelchairs but ignore the regulations of hardware like illumination, color contrast, hearing aid system. America standards have clear requirements for hardware about vision and hearing aid in public buildings. For instance, the hearing aid system 
is clearly defined. This clause applies to areas where hearing is the main mode of communication, such as concert halls, lecture halls, theaters, theaters, conference rooms, etc. Such a public space must be fitted with a qualified listening system, if it can accommodate at least 50 people, or they have an audio amplifier system and have a fixed seat. .American standards refined several different hearing aid systems as applied range of necessary accessibility facilities in buildings. Take museum for example, the hearing aid system installed includes FM broadcast, infrared ray, traditional induction circuit, 3-D loop system and so on.

\section{The reason for those gaps}

Based on the comprehensive analysis of the content of domestic and abroad accessible design, I think the reason why our country is behind the American and European countries in aspect of accessible design standards and constructions mostly is the difference based on values and professional education.

\subsection{The differences in values}

The formation of western countries' values can be traced back to the Renaissance whose guiding ideology idea is humanism. The humanism advocates individual as center, preach individualism and suggest to develop people's ego and self- expression. The disabled and the aged in western countries don't want to be separated from society and treated differently. That require society environment and architecture environment can satisfy their need to exercise alone. In addition, children of America and England usually become fully independent in economical and life aspects. Identically, old parents would not become burden, they choose to live in nursing home and supported by the society. Therefore, the European and American countries embodies the civil rights which means the government serves the public in aspects of laws and welfare policy.

Due to our culture of group and tradition of respecting the old and cherishing the young, we tend to provide special services to the disabled, the aged, and the children and so on. But we usually ignore that the obstacles made by the architecture environment itself can bother the disadvantaged when they participate in society activities individually. In fact, the expense of the construction of accessible design only a little part of construction cost. The ratio of the construction of accessible environment cost to the total cost is approximately $1 \%$, there is no need to add more space to many facilities in the construction if we take the necessary width of the door, the opening direction, the suitable height of working face of various equipment in kitchen, the threshold, the handrail in the bathroom into considerations during early process of design. Otherwise, it will cost a lot to reform the building to suit the requirement of accessible design, and it will also cause the architect and owner to regret the decision. We should see that the improvement of accessibility environment may cost some fund, but it can reduce the fee of social service. Accessibility environment construction has considerable economic benefits from the long, social and general aspects.

\subsection{The differences in professional education}

Nowadays, the education of accessible design in our country is relatively weak, the pertinent researches start and we have few facilities related. In China, except several universities like Tongji University who offered relevant courses, most universities usually referred the practice of accessible design and relevant codes just in curriculum design practice. The depth and popularization are not enough. Our first institute which targets the construction and development of accessibility facilities named as "Joint research center of accessibility construction project" established until May 16, 2011. By contrast, the architecture of EU, America and Japan think highly of accessible design, their research in the field of accessible design has been in the leading position in the world. Many majors in Danish universities offer curriculum related to accessible design, they also hold design competition on the subject of accessible design to promote students learn relevant knowledge further [7]. New York State University started the research about accessible design in 1970s, they have specialized lab and teaching group. There is $70 \%$ of Japanese universities offer curriculum about accessible design [8].

\section{Conclusions}

We should perfect the corresponding codes, standards, and atlas and so on, including dimension markings and atlas illustrated clearly to avoid the ambiguity caused by word expression. The codes should clear the applicable scope of mandatory provisions and main accessibility area in various public buildings. The codes should emphasize the completion of accessible routes to make sure the accessibility environment built is continuous systematic and efficient.

We should increase the investment of new technology and equipment which is helpful to remove the obstacles of vision and hearing. The development of accessibility products should complement current accessibility facilities to remove the obstacles people meet in society when they walking, watching and hearing as far as possible. We should help the handicapped to walk outdoor and join the society.

Government should establish monitoring system about the construction of accessibility environment, including the implement of accessible design, the evaluation and feedback of the environment built already and the maintenance and management measures. European and American public buildings not only have clear and mature technical support but also have the guarantee of stable legislation, judicature and law enforcement system. So, it is important to build excellent accessibility environment judicature guarantee system and carry out the generality of built environment for administration. 


\section{References}

1. Han Ying, Wang Yan, Wang Qiang. Evaluation on Indoor Barrier-free Stream Line of Museum [J]. Architecture and Culture. 2013(10): 81.

2. Premier's Council on the Status of Disabled Persons. Improving Universal Design Requirements in the New Brunswick Building Code [D], 2007.

3. Ministry of Housing and Urban-Rural Development of the People's Republic of China. Code for Accessibility Design[S].Beijing: China Architecture \& Building Press, 2012.

4. $\mathrm{Hu}$ Chuanhai. The Establishment and Implementation of Technical Standards System for
Accessibility Construction in China [DB/OL].http://www.doc88.com, 2013-4.

5. Li Xiuying. An Analysis of Japan 's Aging Society and Its Problems [J].Japan Studies.1989(3): 36.

6. Department of Justice. 2010 ADA Standards for Accessible Design [S]. 2010.

7. Naoto Tanaka, Kunio Hoshiba.Accessible design: design method of five stimulate ways [M].Beijing: China architecture \& building press, 2013: 170.

8. Ecns. Chinese first Joint research center of accessibility construction project established at Tongji

University.[EB/OL]http://edu.ifeng.com/gundong/de tail_2011_05/16/6426685_0.shtml, 2011-05-16. 